\title{
Caracterización Reológica de Pastas untuosas Artesanal y Tecnificada de Ajonjolí (Sesamum indicum) Cultivado en Zambrano-Bolívar (Colombia)
}

\author{
Diofanor Acevedo*, Piedad M. Montero y Yesid A. Marrugo \\ Universidad de Cartagena, Facultad de Ingeniería, Programa de ingeniería de Alimentos, \\ Avenida el Consulado, Calle 30 No. 48-152. Cartagena, Bolívar-Colombia \\ (e-mail: diofanor3000@gmail.com) \\ * Autor a quien debe ser dirigida la correspondencia
}

Recibido Ene. 15, 2014; Aceptado Feb. 12, 2014; Versión final recibida Mar. 4, 2014

\begin{abstract}
Resumen
El objetivo del presente estudio fue evaluar las propiedades reológicas de pastas untuosas artesanal y tecnificada de ajonjolí (Sesamum indicum) cultivado en Zambrano-Bolívar (Colombia). La caracterización reológica de los productos alimenticios es importante en la formulación, procesamiento, transporte y almacenamiento de alimentos, especialmente para emulsiones y suspensiones. El conocimiento de las propiedades viscoelásticas es muy útil en el diseño y predicción de la estabilidad de muestras almacenadas. En este estudio se determinaron los módulos de almacenamiento, el modulo viscoso y el comportamiento de flujo de las pastas de ajonjolí elaboradas, una por método tradicional y otra por un método tecnificado. En la pasta tecnificada la humedad y la sinéresis fueron menores mientras que los porcentajes de carbohidratos, grasa y proteínas fueron mayores. Todas las pastas tuvieron comportamiento pseudoplástico y el carácter elástico predominó sobre el viscoso en la pasta tecnificada.
\end{abstract}

Palabras clave: Sesamum indicum, reología, pasta de ajonjolí, propiedades viscoelásticas

\section{Study of the Rheological Properties of Artisanal and Technified Pastas of oily Sesame (Sesamum indicum) Grown in Zambrano-Bolívar (Colombia)}

\begin{abstract}
The aim of this study was to evaluate the rheological properties of artisanal and technified pastas of oily sesame (Sesamum indicum) grown in Zambrano-Bolívar (Colombia). The rheological characterization of food products is important in the formulation, processing, transportation and food storage, especially for emulsions and suspensions. Knowledge of the viscoelastic properties is useful in the design and prediction of the stability of stored samples. In this study, the viscous modulus and the flow behavior of oily sesame pastes prepared using a traditional artisanal method and a technified method were determined. In the technified pasta moisture and syneresis were lower while the percentages of carbohydrates, fats and proteins were higher. All pastas showed pseudoplastic behavior and the elastic nature predominated over the viscous behavior in the technified pasta.
\end{abstract}

Keywords: Sesamum indicum, rheology, sesame paste, viscoelastic properties 


\section{INTRODUCCIÓN}

El ajonjolí (Sesamum indicum) es una oleaginosa cultivada principalmente en Asia y África (Dada y Adeparusi, 2012; Boureima et al., 2012). Es un importante cultivo de semilla de aceite, debido a sus altos valores nutricionales y terapéuticos, cultivado ampliamente en las regiones templadas tropicales, subtropicales, y en el sur (Hata et al., 2012). La pasta de ajonjolí es un tipo de suspensión coloidal proteínaaceite según Çiftçi et al., (2008), compuesta principalmente de sólidos hidrófilos suspendidos en aceite. Es usada como ingrediente para postres cremosos y salsas. Durante la producción de la pasta las semillas seleccionadas son descascaradas mecánicamente, tostadas y molidas (Çiftçi et al., 2008). La pasta de sésamo es de alto valor nutritivo, pues es rica en lípidos (54-65\%), proteínas (17-27\%), carbohidratos (6,4$21 \%)$, fibra dietética $(9,3 \%)$, niacina $(4,5 \mathrm{mg} / 100 \mathrm{~g})$, tiamina $(1,08 \mathrm{mg} / 100 \mathrm{~g})$ y algunos minerales como: calcio (100 mg/100 g), hierro (9 mg/100 g) y fósforo (807-840 mg/100 g) (Razavi et al., 2007).

La caracterización reológica de los productos alimenticios es importante en la formulación, procesamiento, transporte y almacenamiento especialmente para emulsiones y suspensiones. El conocimiento de las propiedades viscoelásticas es muy útil en el diseño y predicción de la estabilidad de muestras almacenadas (Da Silva et al., 1992). Uno de los métodos frecuentemente usado para caracterizar la viscoelásticidad de los fluidos alimenticios son los ensayos reológicos dinámicos donde se aplica de forma oscilatoria una pequeña deformación o velocidad de deformación sobre un fluido y la amplitud de la respuesta del esfuerzo cortante y del ángulo de fase entre el esfuerzo cortante y la deformación se mide. Este ensayo corresponderá al estado de viscoelasticidad lineal si el esfuerzo es linealmente proporcional a la deformación aplicada y si la respuesta del esfuerzo es en forma de una onda sinusoidal. En el caso de un sólido elástico esta onda está en fase con la velocidad de deformación aplicada. Para un líquido viscoso ideal existe un desfase de $90^{\circ}$ entre ellos. En fluidos viscoelásticos el ángulo de desfase está comprendido entre 0 y $90^{\circ}$ (Steffe, 1996).

En estudios anteriores con pastas de ajonjolí Abu-Jdayil et al., (2002) estudiaron el Tehineh (Pasta de ajonolí), un alimento local en el Oriente Medio, que se produce a partir de semillas de sésamo descascarados. En este estudio, las curvas de flujo y propiedades de flujo en función del tiempo de Tehineh se evaluaron a diferentes temperaturas, encontrando que este se comportaba como producto alimenticio pseudo-plástico no newtoniano, y el modelo de ley de potencia era el más apropiado para adaptarse a las curvas de flujo. Según estos autores una preocupación importante que enfrenta la industria de Tehineh es la producción y el mantenimiento del producto preservando, al mismo tiempo que se desean preservar la consistencia adecuada, la estabilidad, el color, y propiedades de textura, para la cual estos estudios revelan bases específicas para la disminución de estos problemas. Aunque la pasta es estable a las reacciones químicas de deterioro por la actividad antioxidante del sésamo y sesaminol, la inestabilidad coloidal es el principal problema durante el almacenamiento porque produce la sedimentación de las partículas sólidas causando el rechazo por el consumidor (Dada y Adeparusi, 2012; Çiftçi et al., 2008), pero estudios anteriores demuestran que la tecnificación del proceso podrís ayudar a mejorar la estabilidad de la suspensión (Acevedo et al., 2013). De acuerdo a lo anterior el objetivo del presente estudio fue determinar la incidencia de los procesos de elaboración de artesanal y tecnificado, en el comportamiento reológico y estabilidad de pastas de de ajonjolí (Sesamum indicum) cultivado en Zambrano - Bolívar.

\section{MATERIALES Y MÉTODOS}

\section{Elaboración artesanal de la pasta untable}

Se utilizaron $2 \mathrm{Kg}$ de semillas enteras de ajonjolí (S. indicum) adquiridas en el Municipio de Zambrano ubicado en el departamento de Bolívar (Colombia). Se limpiaron y se tostaron en una estufa convencional entre 30 y 35 minutos, hasta obtener un color café claro que indica que el tostado finalizó. Posteriormente las semillas tostadas se molieron dos veces. A la pasta obtenida se le agrega un poco de agua con sal al gusto y se amasó durante10 minutos, hasta obtener la consistencia deseada.

\section{Elaboración tecnológica de la pasta untable}

Se utilizaron $2 \mathrm{Kg}$ de semillas de ajonjolí, adquiridas en el municipio de Zambrano-Bolívar, a los cuales se les realizó limpieza para eliminar impurezas, luego se sumergieron en 6 litros de agua $\left(T=18 \pm 2{ }^{\circ} \mathrm{C}\right)$ durante 12 horas. Las semillas remojadas fueron exprimidas y descascaradas mecánicamente empleando un paño seco para frotar. Luego se sumergieron durante cinco minutos $2 \mathrm{Kg}$ en 8 litros de solución salina al $25 \%$, para separar las cascaras e impurezas que formaban una masa con las semillas. La inmersión de las semillas en la solución salina, se repitió tres veces. Las semillas fueron tomadas desde la superficie de la solución y luego se lavaron con agua cinco (5) veces para eliminar la sal. Posteriormente las semillas se exprimieron para reducir el contenido de agua, luego fueron tostadas. Para el tostado $1 \mathrm{Kg}$ de semillas de 
ajonjolí descortezado húmedo fueron tostadas a temperatura controlada de $130^{\circ} \mathrm{C}$, durante 30 minutos con agitación en un horno marca Challerger. Las semillas tostadas se molieron en un molino para granos marca corona, la molienda se realizó dos veces. La pasta fue mezclada con $0,10 \%$ de sal y homogenizada en un homogeneizador marca Oster, por 2 minutos; obteniendo un producto con consistencia cremosa y sabor definido. La pasta terminada fue envasada en recipientes de vidrio cerrados herméticamente. Se aplicó un tratamiento de pasteurización a $95{ }^{\circ} \mathrm{C}$ por 10 minutos, contados a partir de que el agua comienza a hervir. Las pastas fueron almacenadas a temperatura de refrigeración (Granados et al., 2010).

\section{Análisis químico proximal y medida de la sinéresis de las semillas y pastas de ajonjolí}

El contenido proximal se realizó según el método A.O.A.C (2003). Después de dos días de almacenamiento refrigerado a $4^{\circ} \mathrm{C}$ se agitaron las pastas por 2 minutos a $400 \mathrm{rpm}$ con un agitador magnético y posteriormente se centrifugaron a $5000 \mathrm{~g}$ a $20^{\circ} \mathrm{C}$. La sinéresis se calculó como la cantidad de líquido que se separa del producto, debido a la centrifugación con relación a la masa total de la pasta que fue centrifugada (Acevedo et al., 2010).

\section{Determinaciones reológicas}

Se tomaron tres muestras de $500 \mathrm{~cm}^{3}$ de cada pasta. Las medidas viscoelásticas se realizaron en un reómetro TA AR $1500^{\circledR}$ (TA Instruments Ltda.) utilizando el sensor de platos concéntricos (40 mm steel plate) de acuerdo a metodología de Acevedo et al., (2013).

\section{Análisis de datos}

Se manejó un diseño experimental totalmente aleatorio. Las determinaciones se efectuaron por triplicado y los resultados expresados como la media \pm la desviación estándar, utilizando el programa Graph Padlnstats versión 3.1. Las variables respuesta fueron el contenido de: proteína, grasa, humedad, fibra, carbohidratos, y para el análisis reológicos fueron el coeficiente de consistencia, el índice de flujo, los módulos de almacenamiento y de pérdida.

\section{ANÁLISIS DE RESULTADOS}

\section{Análisis químico proximal de las semillas y pastas de ajonjolí}

Como se observa en la Tabla 1 la pasta artesanal presenta mayor porcentaje de humedad que la tecnificada debido a que después de obtenida se le adiciona agua, este porcentaje puede facilitar su separación en dos fases y disminuir su estabilidad. Mientras que en la pasta tecnificada son mayores los porcentajes de carbohidratos, grasa y proteínas lo que puede contribuir a la estabilidad de la suspensión (Alpaslan y Haita, 2002). Resultado parecido se reportó el trabajo de Acevedo et al., (2013), quienes trabajaron con pasta de ajonjolí artesanal y tecnificada de muestras provenientes del municipio de Córdoba-Bolívar (Colombia).

Tabla 1: Análisis proximal del ajonjolí y de la pasta artesanal y tecnificada.

\begin{tabular}{|c|c|c|c|}
\hline Componentes (\%) & Ajonjolí & Pasta artesanal & Pasta Tecnificada \\
\hline Humedad & $5,98 \pm 0,45$ & $23,31 \pm 1,55$ & $0,62 \pm 0,05$ \\
\hline Proteínas & $18,81 \pm 0,55$ & $20,53 \pm 0,67$ & $23,8 \pm 0,42$ \\
\hline Fibra & $4,06 \pm 0,04$ & $4,1 \pm 0,075$ & $4,76 \pm 0,035$ \\
\hline Cenizas & $0,77 \pm 0,056$ & $0,70 \pm 0,034$ & $0,78 \pm 0,043$ \\
\hline Grasa & $39,64 \pm 0,24$ & $31 \pm 0,99$ & $41,17 \pm 0,85$ \\
\hline Carbohidratos & $30,58 \pm 0,65$ & $20,36 \pm 0,73$ & $28,86 \pm 0,92$ \\
\hline
\end{tabular}

\section{Medida de la sinéresis}

La sinéresis de la pasta artesanal con un $23 \pm 2,09 \%$ fue mayor que la de la pasta tecnificada con solo un $5 \pm 0,42 \%$. Estas diferencias son debidas a que el contenido de humedad en la pasta artesanal es mayor, por lo cual es menos estable, y por tanto con menor vida útil resultados similares presentaron las pastas elaboradas con ajonjolí cultivado en Córdoba Bolívar (Acevedo et al., 2013; 2010). El porcentaje de proteínas y carbohidratos en la pasta tecnificada por su capacidad de retención de humedad contribuyen a la menor sinéresis (AlpaslanyHaita, 2002).

Pruebas dinámicas oscilatorias

Los cambios en los módulos elástico y de pérdida con el aumento de la frecuencia se muestran en las Figura 1 para la pasta artesanal. El módulo de pérdida o viscoso fue mayor que el modulo elástico, porque presenta un comportamiento predominantemente liquido debido al porcentaje de humedad (Razavi et al., 2007; Alpaslan y Haita, 2002). En la Figura 2 el modulo elástico o de almacenamiento es mayor que el 
modulo de pérdida o viscoso para la pasta tecnificada. El alto valor del módulo de almacenamiento es un indicativo de la fuerte interacción partícula - partícula o de una estructura tipo red estabilizada (Çiftçi et al., 2008).

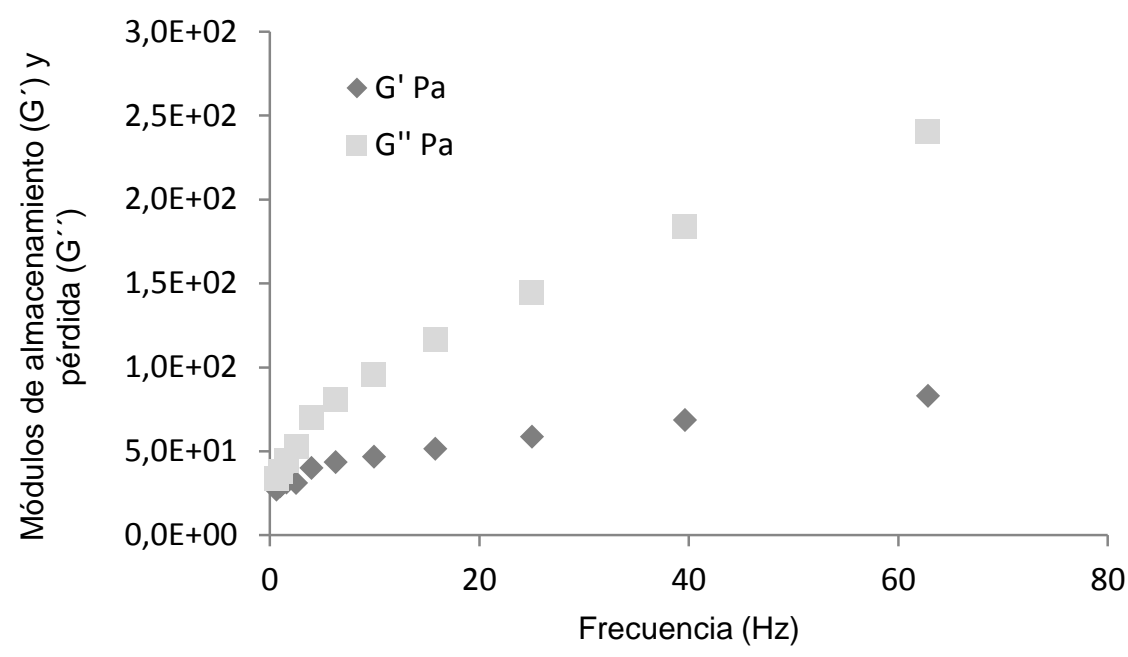

Fig. 1: Módulos de almacenamiento y pérdida de pasta de ajonjolí artesanal

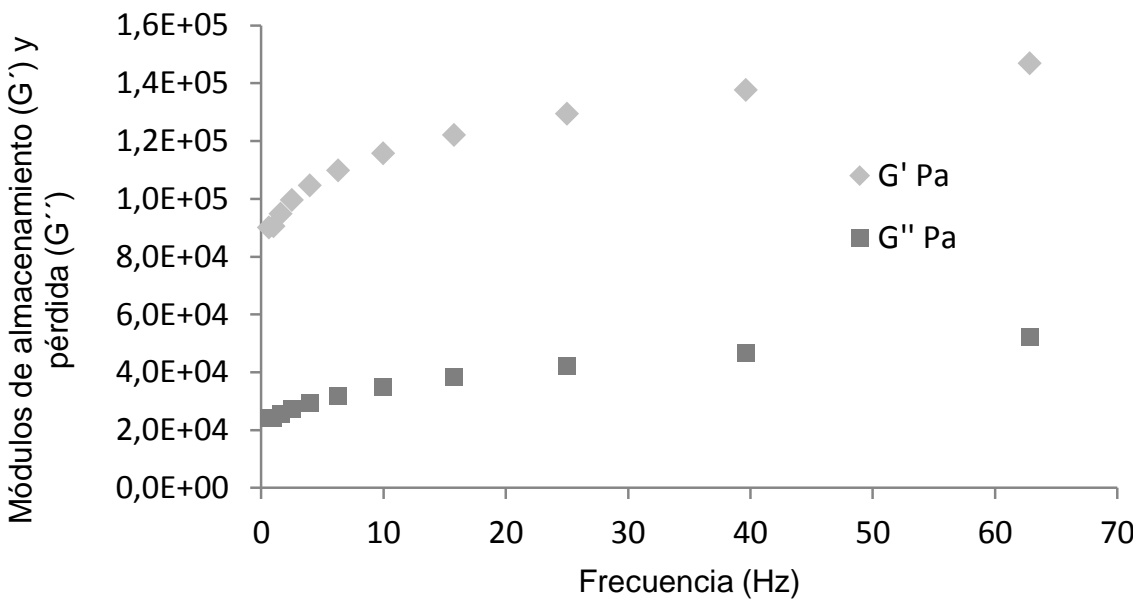

Fig. 2: Módulos de almacenamiento y pérdida de pasta de ajonjolí tecnificada

\section{Comportamiento de flujo}

En la Figura 3 se presentan las curvas de flujo de las pasta de ajonjolí elaboradas artesanal y tecnológicamente. Las curvas de la pasta artesanal y tecnificada presentaron comportamiento pseudoplástico, o de adelgazamiento al corte. Estudios anteriores han informado que la pasta de sésamo, tiene comportamiento pseudoplástico (Çiftçi et al., 2008; Altay y Ak, 2005). Se probaron con los modelos de Herschel-Burkley y la ecuación de la ley de la potencia. El modelo que mejor se ajustó fue el de la ley de potencia presentando el coeficiente de correlación de $(0,987)$. Las propiedades de flujo se ven afectadas significativamente por el porcentaje de agua, la cual produce la disminución del coeficiente de consistencia, mientras aumenta el índice de comportamiento de flujo (Çiftçi et al., 2008).

El comportamiento de flujo por cizalla o adelgazamiento al corte de pasta de sésamo está de acuerdo con los hallazgos de otros trabajos sobre las propiedades reológicas de los productos de pasta de sésamo (AbuJdayil et al., 2002; Alpaslan y Haita, 2002; Arslan et al., 2005; KayayBelibagli, 2002; Sengul et al., 2005). Por ejemplo Arslan et al., (2005) estudiaron las propiedades reológicas de tahin/pekmaz, un tipo de pasta de sésamo, combinada a diferentes concentraciones de Tahin $(20-32 \%)$ y temperaturas de 35 a $65{ }^{\circ} \mathrm{C}$, y encontraron que las mezclas mostraron un comportamiento adelgazamiento por corte en todas las condiciones ensayadas. 


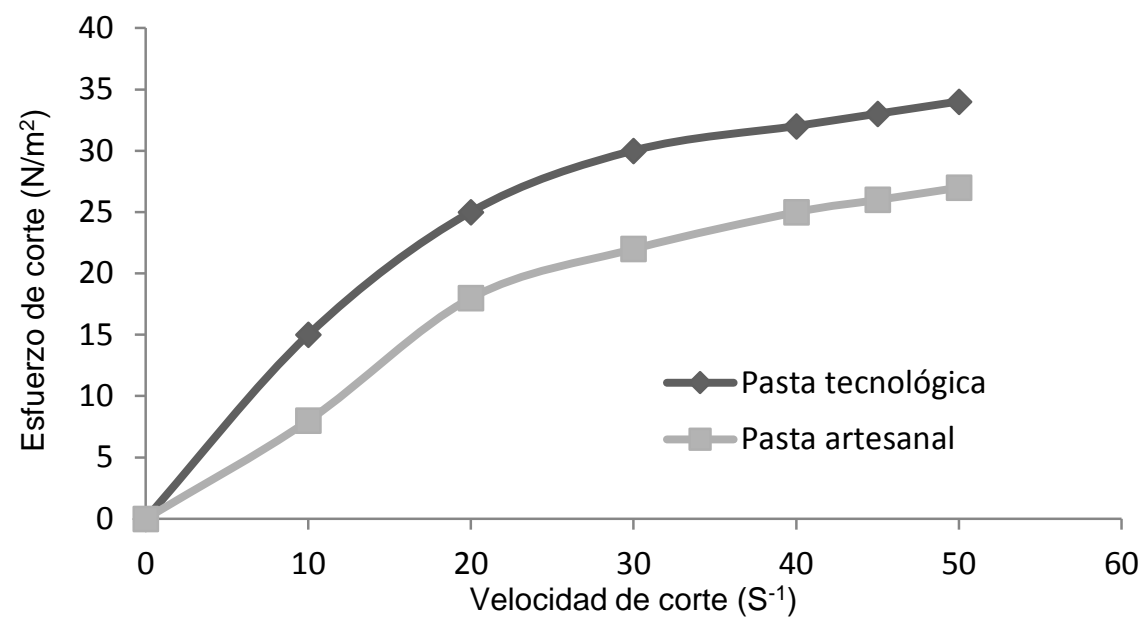

Fig. 3: Esfuerzo de corte en función de la velocidad de corte para la pasta de ajonjolí artesanal y tecnológica.

Tabla 2: Mediciones reológicas de flujo en el modelo de ley de potencia

\begin{tabular}{|l|l|l|l|}
\hline Sistema & $K$ & $n$ & $R^{2}$ \\
\hline Pasta artesanal & $2,943 \pm 0,02$ & $0,784 \pm 0,04$ & $0,987 \pm 0,033$ \\
\hline Pasta tecnológica & $7,133 \pm 0,12$ & $0,4541 \pm 0,06$ & $0,997 \pm 0,011$ \\
\hline
\end{tabular}

En la Tabla 2, se presentan el coeficiente de consistencia $\mathrm{K}$ y el índice de comportamiento de flujo $\mathrm{n}$. Las pasta tecnificada presenta mayor $K$ y menor $n$ que la pasta elaborada artesanalmente esto es debido al bajo contenido de humedad y mayor contenido de carbohidratos lo que hace que presenten mayor resistencia al flujo (Razavi et al., 2007). Cuanto mayor sea el valor de $n$, mayor es el comportamiento newtoniano, es decir más fluida es, tal como pasa con la pasta artesanal (Razavi et al., 2007; Alpaslan y Haita, 2002).El aumento de la velocidad de corte produce un daño irreversible en la estructura de las pastas. Las proteínas fueron el principal componente dañado porque la fase separada de aceite tiene características newtonianas y las viscosidad disminuye, mientras la fase solida, principalmente proteína, tiene características de sólido (Razavi et al., 2007; Çiftçi et al., 2008). Estos resultados coinciden con los reportados por otros autores (Acevedo et al., 2013; Razavi et al., 2007; Mohammad y Alaei, 2006).

\section{CONCLUSIONES}

Los porcentajes de carbohidratos, proteínas y agua influyen en las propiedades reológicas de las pastas de ajonjolí artesanal y tecnificada, y en la sinéresis, la cual fue mayor en la pasta elaborada artesanalmente. Todas las pastas tuvieron comportamiento pseudoplástico. En la pasta tecnificada el carácter elástico es mayor que el viscoso mientras que para la pasta artesanal fue al contrario. Este trabajo demuestra como la elaboración de pastas untuosas de ajonjolí resultan más eficientes mediante un método tecnificado, siguiendo protocolos bajo bases científicas y técnicas.

\section{REFERENCIAS}

Abu-Jdayil, B., K. Al-Malah y H. Asoud, Rheological characterization of milled sesame (tehineh), Food Hydrocolloids: 16, 55-61 (2002).

Acevedo, D., A. Rodríguez, y A. Fernández, Efecto de las Variables de Proceso sobre la Cinética de Acidificación, la Viabilidad y la Sinéresis del Suero Costeño Colombiano, InformaciónTecnológica: 21 (2), 29-36 (2010).

Acevedo, D., P. Montero y Y. Marrugo, Evaluación de las propiedades reológicas de pastas de ajonjolí artesanal y tecnificada, Revista U.D.C.A Actualidad \& Divulgación Científica 16(1): 245-251 (2013).

Alpaslan M. y M. Hayta, Rheological and sensory properties of pekmaz (grape molasses)/tahin (sesame paste) blends, Journal of Food Engineering: 54, 89-93 (2002).

Altay, F.L. y M.M. Ak, Effects of temperature, shear rate and constituents on rheological properties of tahin (sesame paste), Journal of the Science of Food and Agriculture: 85, 105-111 (2005). 
AOAC., Métodos de análisis de la asociación oficial de química analítica para determinar humedad, fibra, cenizas, grasa y proteína., Washington, U.S.A., Chapter 32: 1, 2, 5 y 14 (2003).

Arslan, E., M.E. Yener y A. Esin, Rheological characteristics of tahin/pekmaz (sesame paste/concentrated grape juice) blends, Journal of Food Engineering: 69, 167-172 (2005).

Boureima, S. y otros cuatro autores, Screening for drought tolerance in mutant germ plasm of sesame (Sesamum indicum) probing by chlorophylla fluorescence, Environmental and Experimental Botany: 81, 3743 (2012).

Çiftçi, D. y otros tres autores, Colloidal stability and rheological properties of sesame paste, Journal of Food Engineering: 87(3), 428-435 (2008).

Dada, A.A. y E.O. Adeparusi, Dietary effects of two medicinal plants (Sesamum indicum) and (Croton zambesicus) on the reproductive indices in female African catfish (Clarias gariepinus) broodstock, The Egyptian Journal of Aquatic Research: 38(4), 269-273 (2012).

Da Silva, J.A.L. y M.A: Rao, Viscoelastic properties of food hydrocolloid dispersions, pp. 285-316. In:Rao, M.A., Steffe, J.F. (Eds.), Viscoelastic Properties of Foods. Elsevier, London, UK, (1992).

Elleuch, M. y otros cuatro autores, Quality characteristics of sesame seeds and by-products, Food Chemistry: 103,641-650 (2007).

Granados, C., G. Gonzalo, y D. Acevedo, Tecnificación, caracterización fisicoquímica y microbiológica del queso de capa de Mompox (Colombia), Rev. Bio. Agro: 8(2), 440-450 (2010).

Hata, N. y otros cinco autores, Effect of photoperiod on growth of the plants, and sesamin content and CYP81Q1 gene expression in the leaves of sesame (Sesamum indicumL.), Environmental and Experimental Botany: 75, 212-219 (2012).

Kaya, A. y K.B. Belibagli, Rheology of solid Gaziantep Pekmaz, Journal of Food Engineering: 54, 221-226 (2002).

Mohammad, H. y Z. Alaei, Rheological Properties of Date Syrup/Sesame Paste Blend, World Journal of Dairy \& Food Sciences: 1(1), 01-05 (2006).

Razavi, S.M.A. y otros tres autores, The time independent rheological properties of low fat sesame paste/date syrup blends as a function of fat substitutes and temperature, Food Hydrocolloids: 21(2), 198-202 (2007).

Sengul, M., M. Fatih Ertugay y M. Sengul, Rheological, physical, and chemical characteristics of mulberry pekmaz, Food Control: 16, 73-76 (2005).

Steffe,J., Rheological methods in Food process engineering, Second Edition, pp. 325- 331. Freeman Press, East Lansing, USA (1992). 